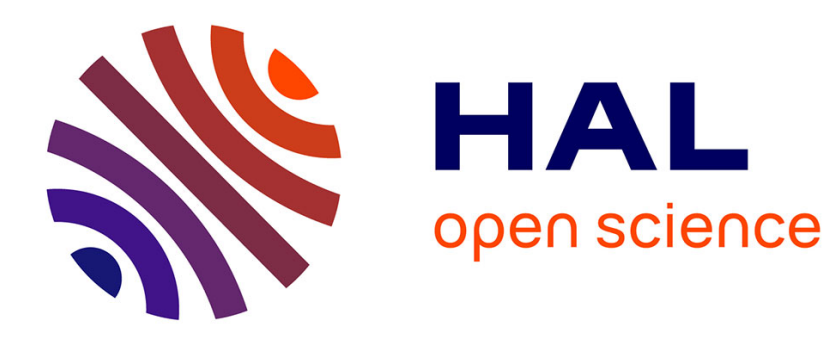

\title{
Dépérissement des chênes et pathogènes
}

Claude Delatour

\section{To cite this version:}

Claude Delatour. Dépérissement des chênes et pathogènes. Revue forestière française, 1990, 42 (2), pp.182-185. 10.4267/2042/26059 . hal-03425033

\section{HAL Id: hal-03425033 \\ https://hal.science/hal-03425033}

Submitted on 10 Nov 2021

HAL is a multi-disciplinary open access archive for the deposit and dissemination of scientific research documents, whether they are published or not. The documents may come from teaching and research institutions in France or abroad, or from public or private research centers.
L'archive ouverte pluridisciplinaire HAL, est destinée au dépôt et à la diffusion de documents scientifiques de niveau recherche, publiés ou non, émanant des établissements d'enseignement et de recherche français ou étrangers, des laboratoires publics ou privés. 


\title{
DÉPÉRISSEMENT DES CHÊNES ET PATHOGĖNES
}

\author{
C. DELATOUR
}

Au cours des années récentes, les "dépérissements" ont pris une place grandissante dans les préoccupations des forestiers: dépérissements du Chêne, du Sapin, de l'Alisier...

Bien qu'appliqué à des réalités concrètement observées sur le terrain, le terme même de " dépérissement " peut paraître ambigu.

Une assez bonne définition a été fournie par Métro (1975) : "dépérissement terminal : désordre progressif plus ou moins rapide d'une cime, du haut vers le bas et de l'extérieur vers l'intérieur, d'où peut résulter la mort d'une partie ou de la totalité de l'arbre ". Plus récemment (DSF, 1988), il a été indiqué que "le terme de dépérissement recouvre implicitement une notion de détérioration de l'état de santé au cours du temps (...), il y a dépérissement si on observe une détérioration prolongée de l'apparence et de la croissance des arbres".

II est donc clair que "dépérissement" est un terme de symptomatologie, c'est-à-dire qu'il caractérise un ensemble d'anomalies perceptibles à l'œil sur le terrain. Ces anomalies correspondent à l'idée que l'on se fait d'une détérioration globale de la santé de l'arbre : réduction de la qualité et de la quantité du feuillage ou des pousses mais surtout mortalité d'organes existants (rameaux en particulier). Le terme inclut aussi une certaine idée d'évolution dans le temps qui traduit le fait que l'issue naturelle pour l'arbre dépérissant est jugée problématique mais pas obligatoirement fatale.

Le dépérissement, dont le constat est en fait basé sur un ensemble de symptômes variables selon les cas, ne suppose pas en lui-même de causes particulières et ne doit donc pas a priori être considéré comme une maladie. En effet, une maladie au sens strict est provoquée par un agent biologique dont l'activité pathogène entraîne à elle seule chez le végétal des désordres reproductibles expérimentalement. Certaines maladies conduisent en fait à des faciès de dépérissement mais, dans ces cas, l'origine doit en être précisée, par exemple "le dépérissement du Cyprès à Seiridium cardinale". Dans bien des cas cependant, le terme trop général de dépérissement sera évité pour désigner une maladie identifiẻe: graphiose de l'Orme, chancre coloré du 
Platane, maladie de l'écorce du Hêtre, flétrissement américain du Chêne... Ainsi, le terme de dépérissement correspond aussi à l'idée d'une cause non identifiée ou non immédiatement identifiable avec certitude.

C'est bien comme cela que s'est présenté le dépérissement des Chênes survenu en France en 1976-1982 (Buffet, 1983 ; Macaire, 1984). Les travaux réalisés à cette occasion ont donné lieu à de nombreux articles dont beaucoup ont été publiés dans la Revue forestière française en 1983.

Les observations réalisées dans les Pyrénées-Atlantiques (Durand et al., 1983) et surtout à Tronçais (Becker, Lévy, 1983; Guillaumin et al., 1983), ainsi que l'analyse de la bibliographie antérieure ont permis d'aboutir clairement aux conclusions suivantes :

- Les dépérissements massifs de Chênes se développent périodiquement en Europe. Ils surviennent brusquement et s'estompent en peu d'années. Des dépérissements plus chroniques sont cependant observés çà et là et sont souvent mentionnés dans certains pays de l'Est et Sud-Est de l'Europe.

- Ils touchent en priorité et, le plus souvent, exclusivement le Chêne pédonculé (Quercus robur); ce sont essentiellement les arbres adultes qui sont concernés (vers 100 ans et au-delà).

- Les dépérissements sont presque toujours précédés ou coïncident avec des périodes où la pluviométrie est particulièrement déficitaire.

- Les sécheresses exceptionnelles ne conduisent cependant pas toujours ni partout au dépérissement. Son apparition est largement conditionnée par de nombreux autres facteurs: facteurs permanents (réserve en eau, richesse des sols, topographie, état sanitaire des racines...) ou facteurs conjoncturels (insectes défoliateurs, oïdium...). Cet ensemble de facteurs, dont certains peuvent avoir des effets drastiques, permet de concevoir que le risque d'apparition du dépérissement est variable.

Comme nous venons de le mentionner, des champignons pathogènes figurent parmi les facteurs qui contribuent au risque de dépérissement. Dans le concert des observations évoquées précédemment, les pathologistes ont montré que les agents de pourridié, Armillaria mellea et proba-

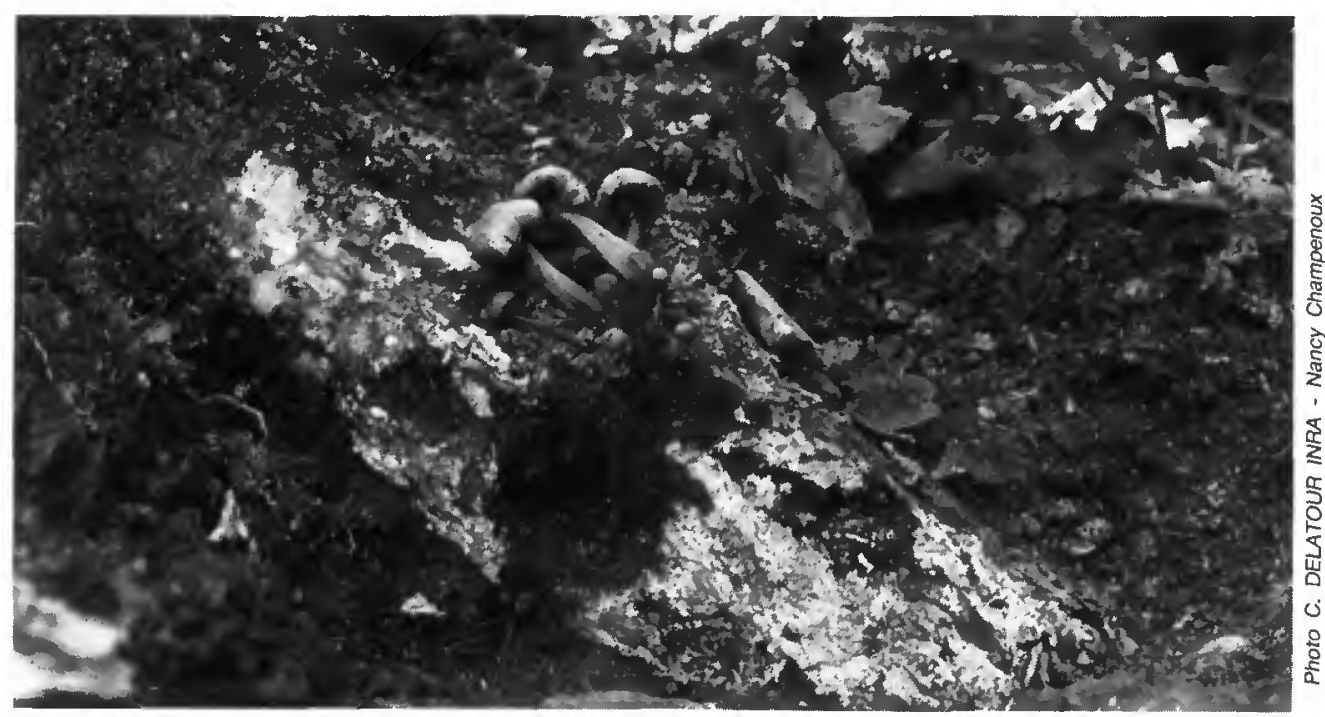

La Collybie peut occasionner de graves lésions aux racines principales des chênes et conduire à terme au dépérissement de l'arbre. (Chêne pédonculé, forêt d'Amance, 54) 
blement plus encore Collybia fusipes (Delatour, Guillaumin, 1984; Guillaumin et al., 1983) peuvent être responsables de dégradations très intenses du système racinaire des chênes, sans aucun symptôme aérien en conditions normales. Ce phénomène reste très peu connu du fait des difficultés d'étude et ne porte pour l'instant que sur l'analyse de cas peu nombreux. Nous pensons cependant que son importance est sous-estimée alors que diverses conséquences semblent pouvoir en résulter. Celles-ci sont schématisées dans la figure 1 (ci-dessous) où il apparaît que, outre les mortalités directes ou indirectes, on peut envisager d'imputer aux pourridiés des effets sur le statut social des arbres. De tels effets sont observés chez Quercus rubra sous l'action de la Collybie, dans le cas de la glandée d'Amérique au domaine des Barres où des arbres dominants présentent des pertes anormales de vigueur.

Ainsi, l'activité permanente des champignons pathogènes au niveau des racines pourrait avoir des effets tout au long de la vie des chênaies alors que les dépérissements spectaculaires n'en seraient qu'une expression extrême rendue possible par des stress exceptionnels.

Un autre groupe de champignons peut être pris en considération dans le cadre du dépérissement du Chêne. II s'agit des Ophiostomatales (Ophiostoma et Ceratocystis) dont certaines sont considérées comme de véritables pathogènes responsables de maladies vasculaires chez les Chênes dans divers pays de l'Est et Sud-Est de l'Europe. Nous avons antérieurement analysé cette question d'un point de vue bibliographique sans pouvoir en tirer de conclusions définitives.

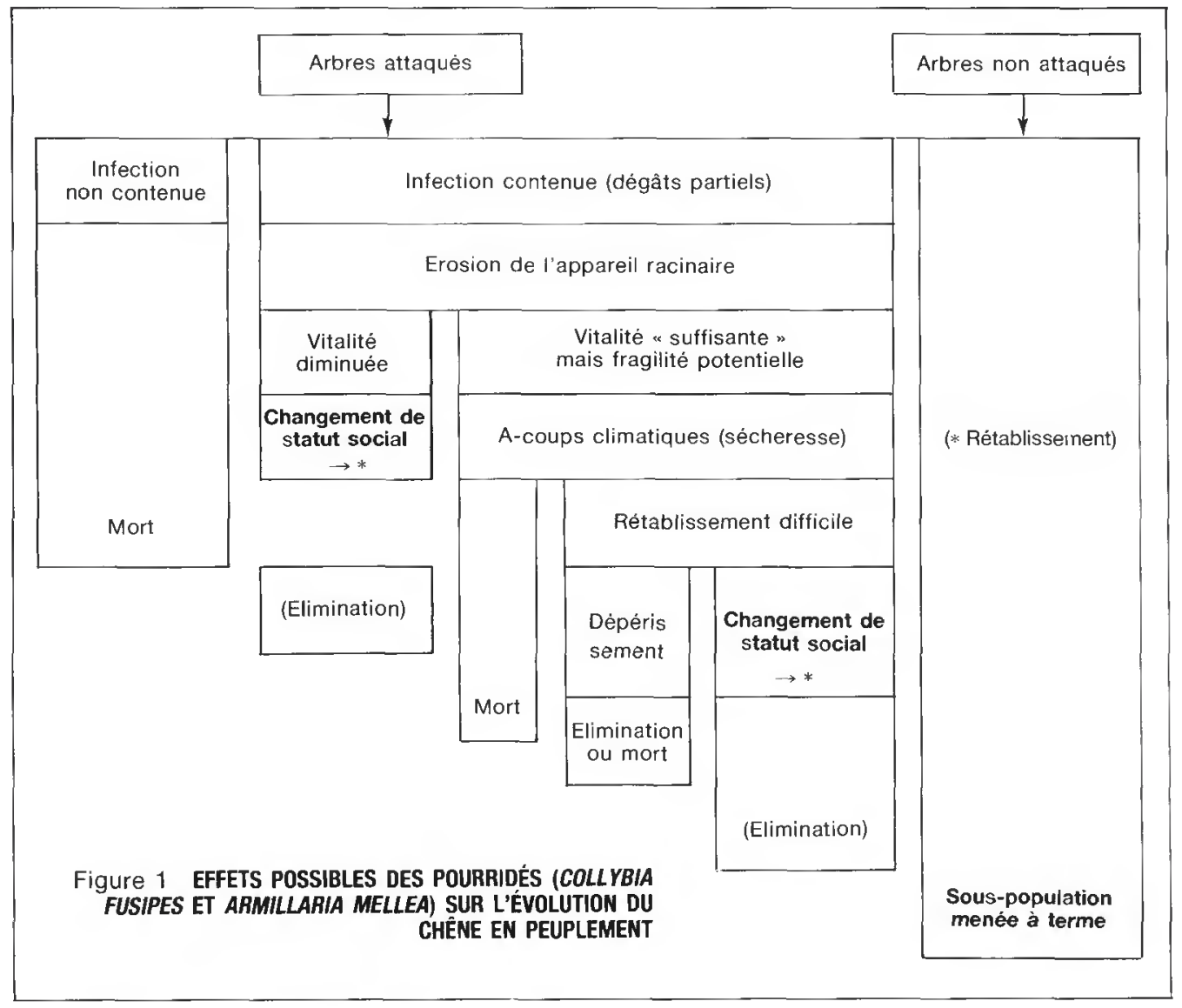


Compte tenu des incertitudes qui règnent en la matière et de l'enjeu potentiel pour nos chênaies, nous avons entrepris de clarifier la situation tant en ce qui concerne la définition des espèces mises en cause que de leur pouvoir pathogène.

Dans ce but, notre Laboratoire a et continue de rassembler le maximum de représentants de ce groupe, en provenance de l'ensemble de l'Europe, et procède à leur caractérisation morphologique afin de savoir si des espèces différentes sont réellement prèsentes dans l'Est de l'Europe. Conjointement, le pouvoir pathogène est testè par inoculation mais en nous limitant, dans ce cas, exclusivement aux isolats ouest-européens. Un lien entre ces deux approches est recherché en ètudiant l'éventuelle phytotoxicité pour le Chêne des filtrats de culture, phénomène parfois cité pour les Ophiostomatales en Europe de l'Est en relation avec des symptômes de flétrissement.

$$
*_{* *}^{* *}
$$

Les travaux de pathologie, menés depuis une dizaine d'années à l'occasion des dépérissements du Chêne ainsi que du Sapin, nous ont conduit à explorer cette partie de l'arbre particulièrement méconnue car difficile d'accès qu'est l'appareil racinaire adulte. Sans doute n'avons-nous qu'entrevu l'importance de certains phénoménes pathologiques à ce niveau, sans pouvoir objectivement en èvaluer la portèe gènérale.

Mais la chênaie française reste un enjeu économique de première importance et son équilibre à long terme mérite une attention soutenue. C'est dans cet esprit qu'est abordée dans notre Laboratoire la question des risques sanitaires potentiels, comme celui du flétrissement nordaméricain et celui moins bien cerné des Ophiostomatales de l'Est de l'Europe.

\section{DELATOUR}

Laboratoire de Pathologie forestière

CENTRE DE RECHERCHES FORESTIÉRES (INRA)

CHAMPENOUX 54280 SEICHAMPS

\section{BIBLIOGRAPHIE}

BECKER (M.), LÉVY (G.). - Le Dépérissement du Chêne, les causes écologiques. Exemple de la forêt de Tronçais et premières conclusions, - Revue forestière française, vol. XXXV, $n^{\circ} 5,1983, p p .341-356$.

BUFFET (M.). - Le Dépérissement du Chêne en forêt soumise. - Revue forestière française, vol. $X X X V, n^{\circ} 3$, 1983, pp. 199-204.

DELATOUR (C.). - Les Dépérissements de Chênes en Europe. - Revue forestière française, vol. $X \times X V, n^{\circ} 4$, 1983, pp. 265-282.

DELATOUR (C.). - Le Problème des Ceratocystis européens des Chênes. - Bulletin OEPP, $\mathrm{n}^{\circ} 16,1986$, pp. 521-525.

DELATOUR (C.), GUILLAUMIN (J.-J.). - Un pourridié méconnu : le Collybia fusipes. - Comptes rendus des Scèances de l'Académie d'Agriculture de France, vol. 70, nº 1, 1984, pp. 123-126.

DÉPARTEMENT DE LA SANTÉ DES FORÊTS. - Santė des Forêts 1988. - Paris : Ministère de l'Agriculture et de la Forêt, 1988. - $65 \mathrm{p}$.

DURAND (P.), GELPE (J.), LEMOINE (B.), RIOM (J.), TIMBAL (J.). - Le Dépérissement du Chêne pédonculé dans les Pyrénèes-Atlantiques. - Revue forestière française, vol. XXXV, ${ }^{\circ} 5,1983$, pp. 357-368.

GUILLAUMIN (J.-J.), BERNARD (Ch.), DELATOUR (C.), BELGRAND (Micheline). - Dépèrissement du Chêne à Tronçais : pathologie racinaire. - Revue forestière française, vol. XXXV, ${ }^{\circ} 6,1983, p p .415-424$.

MACAIRE (A.). - Le Dépérissement du Chêne pédonculé en forêt communale d'Amance (Aube). - Revue forestiere française, vol. XXXVI, $n^{\circ} 3,1984$, pp. 201-205.

METRO (A.). - Dictionnaire forestier multilingue. - Conseil international de la Langue française, 1975. $432 \mathrm{p}$. 